\title{
Errata: One-Dimensional Caricature of Phase Transition ${ }^{1}$ Roberto H. Schonmann ${ }^{2}$ and Nelson I. Tanaka ${ }^{3}$
}

Received February 26, 1991

This paper appeared with some important misprints because the galley proofs were apparently lost in the mail. The main corrections are the following.

p. 244 , line 6 :

p. 246, line 13:

p. 246, line 14:

p. 246, line 5 from bottom: $E(\cdot)$ instead of $R(\cdot)$

p. 247 , line 9 from bottom: $\zeta_{\beta}$ instead of $\xi_{\beta}$

p. 247 , line 3 from bottom: $|x|>\tau$ instead of $|x| \neq \tau$

p. 248 , line 10 :

p. 248 , line 16 :

p. 248 , line 17 :

$$
\begin{aligned}
& m N^{1 / 2-1 / 2 \alpha} \text { instead of } m N^{1 / 2}\{1 / 2 \alpha \\
& X_{l}=I^{2 l-1}-I^{2 l-2} \text { instead of } \\
& X_{1}=I^{2 l}-1-I^{2 l-2 X} \\
& I^{2 l}-I^{2 l-1} \text { instead of } I^{2 l}-I^{2 l}-1
\end{aligned}
$$

p. 248 , line 6 from bottom:

0 instead of $A 0$

$$
2(1-p)^{j \tau N^{1 / 2+1 / 2 x}}
$$

$$
\text { instead of } 2(1-p) j \tau N^{1 / 2+1 / 2 \alpha}
$$

$\exp \left(-j \tau N^{1 / 2+1 / 2 x} p\right)$ instead of $\exp \left(j \tau N^{1 / 2+1 / 2 x} p\right)$

p. 249 , line 2 from bottom: $\leqslant P(\ldots)$ instead of $\leqslant(\ldots)$

p. 250, line 11:

$$
P\left(\max _{s=1, \ldots, k}\left|\frac{\sum_{i=1}^{s}\left(U_{i}-V_{i}\right)}{N^{1 / 2+1 / 2 \alpha}}\right|>\varepsilon / 2\right)+P\left(\max _{i=1, \ldots, k+1}\left|\frac{U_{i}}{N^{1 / 2+1 / 2 \alpha}}\right|>\varepsilon / 2\right)
$$

instead of

$$
P\left(\max _{s=1, \ldots, k}\left|\frac{U_{i}}{N^{1 / 2+1 / 2 \alpha}}\right|>\varepsilon / 2\right)
$$

p. 250 , line 2 from bottom: $(\alpha+3)$ instead of $(\alpha++3)$

\footnotetext{
${ }^{1}$ This paper originally appeared in J. Stat. Phys. 61:241-252 (1990).

${ }^{2}$ Mathematics Department, UCLA, Los Angeles, California 90024.

${ }^{3}$ IME-USP, Caixa Postal 20570, 01498 Sao Paulo SP, Brazil.
} 